\title{
Numerical Solution of Oscillatory Motion of Dusty Visco-Elastic Fluid Through Porous Media
}

\author{
Dr. Rafiuddin ${ }^{1}$ and M.V.Ramana Murthy ${ }^{2}$ \\ ${ }^{1}$ CVR College of Engineering, Department of H \& S, Ibrahimpatan, R.R.District, A.P., India \\ Email: rafiuddin2008@gmail.com \\ ${ }^{2}$ Osmania University, Department of Mathematics, Hyderabad, India \\ Email:mv_rm@rediffmail.com
}

\begin{abstract}
The solution of oscillatory motion of dusty viscoelastic fluid through porous media by finite element method is obtained; expressions for the velocity of fluid, dust and skin friction are also obtained. The effects of various parameters on above are shown graphically and discussed.
\end{abstract}

Index Terms - Oscillatory motion, Dusty - Water's liquid B, Porous medium.

\section{INTRODUCTION}

Dusty fluids find applications in such diverse fields as the transportation of sediment by water and air, the centrifugal separation of particulate matter from fluids, fluid-droplet sprays, dust collection, nuclear reactor cooling, acoustics, environmental pollution, batch settling, rain erosion and guided missiles, among others.

The constitutive equation for the rheological equations of state for a memory fluid (Walter's liquid B model) given by Walter (1960, 1962).Grover (1968) studied the motion of an incompressible viscous fluid bounded by two infinite plates, the upper one is fixed and the other executing a simple harmonic oscillation in its own plane. Siddappa and Shanker Hegde (1972) have extended the Grover's work for oscillatory motion of visco-elastic fluid given by Rivlin-Ericksen constitutive equation. Sattar and Ahmed (2007) obtained the numerical solution of Non-Newtonian fluid. Rafiuddin et al. (2006) derived the exact solution of oscillatory motion of a memory fluid through porous media with a horizontal force. Ramu et al. (2010) presented the numerical solution of the above problem.

The aim of the present investigation is to study oscillatory motion of dusty visco-elastic fluid (Walter's liquid $\mathrm{B}^{\prime}$ model) through porous medium which is bounded by two infinite parallel plates.

\section{FORMULATION OF THE PROBLEM}

Consider the oscillatory motion of a dusty visco-elastic fluid bounded by two infinite parallel plates through a porous medium. Let the direction of motion of the fluid be along the $\mathrm{x}$-axis, which is chosen along the lower plate and the $y$-axis be perpendicular to it. Let $(\mathrm{u}, \mathrm{v}, \mathrm{w})$ be the velocity components of the fluid. For the present study, $v$ $=\mathrm{w}=0$. The velocity of the oscillating plate at any instant is taken as $u=\operatorname{Re}\left(u_{0} e^{-i \omega t}\right)$, where 'Re' represents the real part. For convenience we drop the expression
'Re', but we take the real part of the final result. The equation of motion governing the dusty visco-elastic flow is of the form

$\frac{\partial u}{\partial t}=\alpha \frac{\partial^{2} u}{\partial y^{2}}+\frac{K_{n}}{\rho}\left(v_{p}-u\right)-\rho \frac{\partial}{\partial t}\left(\frac{\partial^{2} u}{\partial y^{2}}\right)-\frac{\alpha u}{\gamma}$

$$
\frac{\partial v_{p}}{\partial t}=\frac{K}{m}\left(u-v_{p}\right)
$$

and the equation of continuity is

$$
\partial \mathrm{u} / \partial \mathrm{x}=0
$$

The equation will now be made dimensionless by introducing the non-dimensional quantities.

$$
\begin{aligned}
& y^{x}=\frac{y}{y_{0}}, \quad u^{x}=\frac{u y_{0}}{\alpha}, \quad t^{\prime}=\frac{\alpha t}{y_{o}{ }^{2}}
\end{aligned}
$$

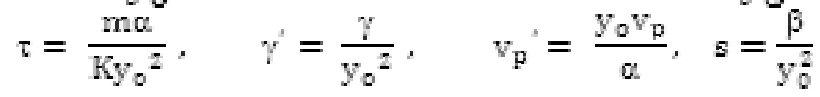

Substituting in (2.1) \& (2.2) dropping the dashes for simplicity, we get

$$
\begin{gathered}
\frac{\partial \mathrm{u}}{\partial \mathrm{t}}=\frac{\partial^{2} \mathrm{u}}{\partial \mathrm{y}^{2}}-\left(\frac{l}{\mathrm{\tau}}+\frac{1}{\gamma}\right) \mathrm{u}-\mathrm{s} \frac{\partial}{\partial \mathrm{t}}\left(\frac{\partial^{2} \mathrm{u}}{\partial \mathrm{y}^{2}}\right)+\frac{\mathrm{lv}_{\mathrm{F}}}{\mathrm{\tau}} \\
\tau \frac{\partial \mathrm{v}_{\mathrm{p}}}{\partial \mathrm{t}}=\left(\mathrm{u}-\mathrm{v}_{\mathrm{p}}\right)
\end{gathered}
$$

Eliminating $\mathrm{v}_{\mathrm{p}}$ from (2.5) making use of (2.6), we get

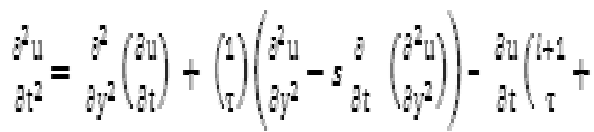

$$
\begin{aligned}
& \frac{1}{y}-s \frac{t^{2}}{\partial t^{2}}\left(\frac{\partial^{2} u}{\partial y^{2}}\right)-\frac{u}{\tau}
\end{aligned}
$$


Where

K- stock's coefficient of resistance $(6 \pi \mathrm{a} \mu)$ for spherical dust particles,

a - average radius of dust particle,

$\mu$ - viscosity of the fluid,

$l-\mathrm{mN}_{\mathrm{o}} / \rho$ mass concentration of dust particle,

$\rho$ - density of fluid,

$\mathrm{m}$-average mass of dust particle,

$\mathrm{N}_{\mathrm{o}}$ - number of dust particle per unit volume,

$\beta$-kinematic visco-elasticity

$\tau-\mathrm{m} / \mathrm{K}$ relaxation time,

$\alpha$ - kinematic viscosity,

$\gamma$-permeability parameter,

$\mathrm{y}_{\mathrm{o}}$-characteristic velocity,

$\mathrm{s}-$ visco-elastic parameter.

\section{SOLUTION OF THE PROBLEM}

Let the lower plate execute simple harmonic oscillations in its own plane whereas the upper plate is fixed .In this case the boundary conditions are

$\mathrm{y}=0, \quad \mathrm{u}=\mathrm{u}_{\mathrm{o}} \mathrm{e}^{-\mathrm{i} \omega \mathrm{t}}$,

$\mathrm{y}=2 \mathrm{y}_{\mathrm{o}}, \quad \mathrm{u}=0$

Where $2 y_{0}$ is the clearance distance between the vibrating plate and fixed plate.

Introducing dimensionless frequency $\omega^{\prime}$ given by $\omega^{\prime}=$ $\omega \mathrm{y}_{\mathrm{o}}{ }^{2} / \alpha$ and using (2.4), the boundary conditions in (3.1) in dimensionless form reduces to

$$
\begin{array}{ll}
\mathrm{y}=0, & \mathrm{u}=\mathrm{e}^{-\mathrm{i} \omega \mathrm{t}}, \\
\mathrm{y}=2, & \mathrm{u}=0,
\end{array}
$$

To solve the equation (2.5), we assume the solution of the form

$u=f(y) e^{-i \omega t}$

Now applying the boundary conditions (3.2) to (3.3), we get
$\mathrm{y}=0$,
$\mathrm{f}(\mathrm{y})=1$,
$\mathrm{y}=2$,
$\mathrm{f}(\mathrm{y})=0 \quad(3.4)$

Substituting (3.3) into (2.5), we have

$\mathrm{f}^{\prime \prime}(\mathrm{y})+\mathrm{mf}(\mathrm{y})=0$

Where

$m=\frac{\left(-1+\omega^{2} w\right)+i \omega[(\alpha+1) y+\tau]}{y\left[\left(1+\omega^{2} r \omega\right)-i \omega(\tau-s)\right]}$

The equation (3.5) is an ordinary differential equation with boundary conditions (3.2) through finite element method using Galerkin method, the solution of (3.5) is given by

$$
f(y)=\frac{1}{2}(2-y)+a\left(y^{2}-2 y\right)
$$

The velocity distribution is given by (3.3)

The real part of the velocity of the fluid is given by

$$
\begin{gathered}
u(y)=\left[\frac{1}{2}(2-y)+C_{1}\left(y^{2}-2 y\right)\right] \cos \omega t+ \\
D_{1}\left(y^{2}-2 y\right) \sin \omega t
\end{gathered}
$$

Skin friction at the lower plate is given by

$$
\begin{gathered}
c_{p}=\left(\frac{\partial u}{\partial y}\right)_{y=0}=-\left(\frac{1}{2}+2 C_{1}\right) \cos \omega t- \\
2 D_{1} \sin \omega t
\end{gathered}
$$

Dust velocity is given by

$$
\begin{gathered}
V_{p}=\frac{\tau}{l}\left[\operatorname { s i n } \omega t \left(-\omega\left(1-\frac{y}{y}\right)-C_{1} \omega\left(y^{2}-2 y\right)-2 D_{1}-\right.\right. \\
\left.2 C_{1} s \omega+\left(\frac{1}{\tau}+\frac{1}{\gamma}\right)\left(y^{2}-2 y\right) D_{1}\right\}+\cos \omega t\left\{D _ { 1 } \omega \left(y^{2}-\right.\right. \\
2 y)-2 C_{1}+2 D_{1} s \omega+\left(\frac{1}{\tau}+\frac{1}{y}\right)\left\{\left(1-\frac{y}{2}\right)+\left(y^{2}-\right.\right. \\
\left.\left.\left.2 y) C_{1}\right)\right\}\right]
\end{gathered}
$$

$$
\begin{gathered}
C_{q}=\left(\frac{\partial V_{y}}{\partial y}\right)_{y=0}=\frac{z}{l}\left[\operatorname { s i n } \omega t \left\{\omega\left(2 C_{1}+\frac{1}{2}\right)-\right.\right. \\
\left.2 D_{1}\left(\frac{l}{\tau}+\frac{1}{\gamma}\right)\right\}-\cos \omega t\left\{\left(\frac{l}{\mathrm{t}}+\frac{1}{\gamma}\right)\left(\frac{1}{2}+2 C_{1}\right)+\right. \\
\left.\left.2 D_{1} \omega\right)\right]
\end{gathered}
$$

Where the constants are not given for the sake of brevity

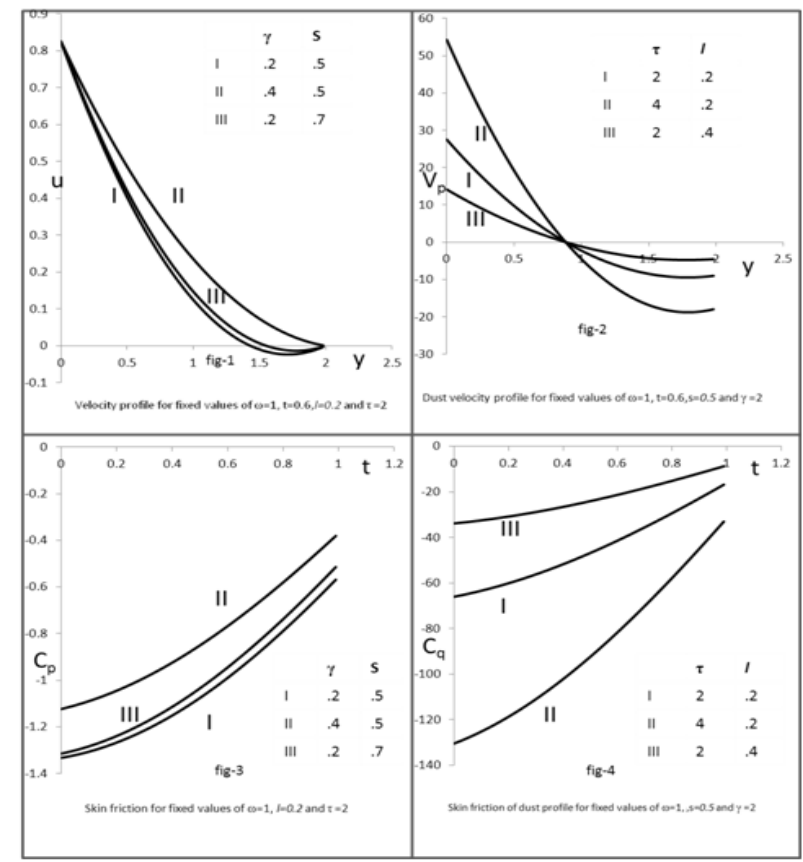

\section{DISCUSSION AND CONCLUSIONS}

From fig-1 it is found that as permeability parameter $(\gamma)$ and visco-elastic parameter (s) increase, fluid velocity (u) increases and from fig-2 we see that mass concentration $(l)$ increases dust velocity $\left(\mathrm{V}_{\mathrm{p}}\right)$ decreases and $\mathrm{V}_{\mathrm{p}}$ increases with relaxation time $(\tau)$, from the middle of the plates the trend is opposite for both. From fig-3 it is observed that skin friction $\left(\mathrm{C}_{\mathrm{p}}\right)$ increases with permeability parameter and visco-elastic parameter. Skin friction in fig- 4 for the dust case $\left(\mathrm{C}_{\mathrm{q}}\right)$ increases with mass concentration and decreases with relaxation time. 


\section{REFERENCES}

[1] Walter, K. Quart. Jour. Mech. Applied Math. 13, pp. 444, 1960.

[2] Walter, K. Quat. Jour. Mech Applied Math. 15 pp. 136, 1962.

[3] Grover, G.K Proc. Int. Soc. Theoretical and Applied Mechanics pp.89, 1968.

[4] Siddappa, B. and Shanker Hegde Progress of Mathematics B.H.U., 6 (2):pp15, 1972.

[5] Rafiuddin, M.V. Ramana Murthy, Mohd. Abdul Rahim and S. Thiagarajan Bulleting of Pure and Applied Sciences, Vol.25E ( 2), pp 1-10, 2006

[6] Arjumand, S. and Ahmed Waheedullah Journal of pure and applied physics vol. 19 No.2 (April- June) pp. 69-75, 2007.

[7] Addepalli Ramu, M.V. Ramana Murthy, Rafiuddin, Arjumand Sattar and Adeeb Nazia Atti Della Fondazione Giorgio Ronchi sept-oct; LXV 5, pp. 607-618, 2010. 